\title{
Low oxygen alters mitochondrial function and response to oxidative stress in human neural progenitor cells
}

Yury M Lages, Juliana M Nascimento, Gabriela A Lemos, Antonio Galina, Leda R. Castilho, Stevens K Rehen

Oxygen concentration should be carefully regulated in all living tissues, beginning at the early embryonic stages. Unbalances in oxygen regulation can lead to cell death and disease. However, to date, few studies have investigated the consequences of variations in oxygen levels for fetal-like cells. Therefore, in the present work, human neural progenitor cells (NPCs) derived from pluripotent stem cells grown in $3 \%$ oxygen (v/v) were compared with NPCs cultured in $21 \%(\mathrm{v} / \mathrm{v})$ oxygen. Low oxygen concentrations altered the mitochondrial content and oxidative functions of the cells, which led to improved ATP production, while reducing generation of reactive oxygen species (ROS). NPCs cultured in both conditions showed no differences in proliferation and glucose metabolism.

Furthermore, antioxidant enzymatic activity was not altered in NPCs cultured in 3\% oxygen under normal conditions, however, when exposed to external agents known to induce oxidative stress, greater susceptibility to DNA damage was observed. Our findings indicate that the management of oxygen levels should be considered for in vitro models of neuronal development and drug screening. 


\section{Low oxygen alters mitochondrial function and response to oxidative stress in human neural progenitor cells}

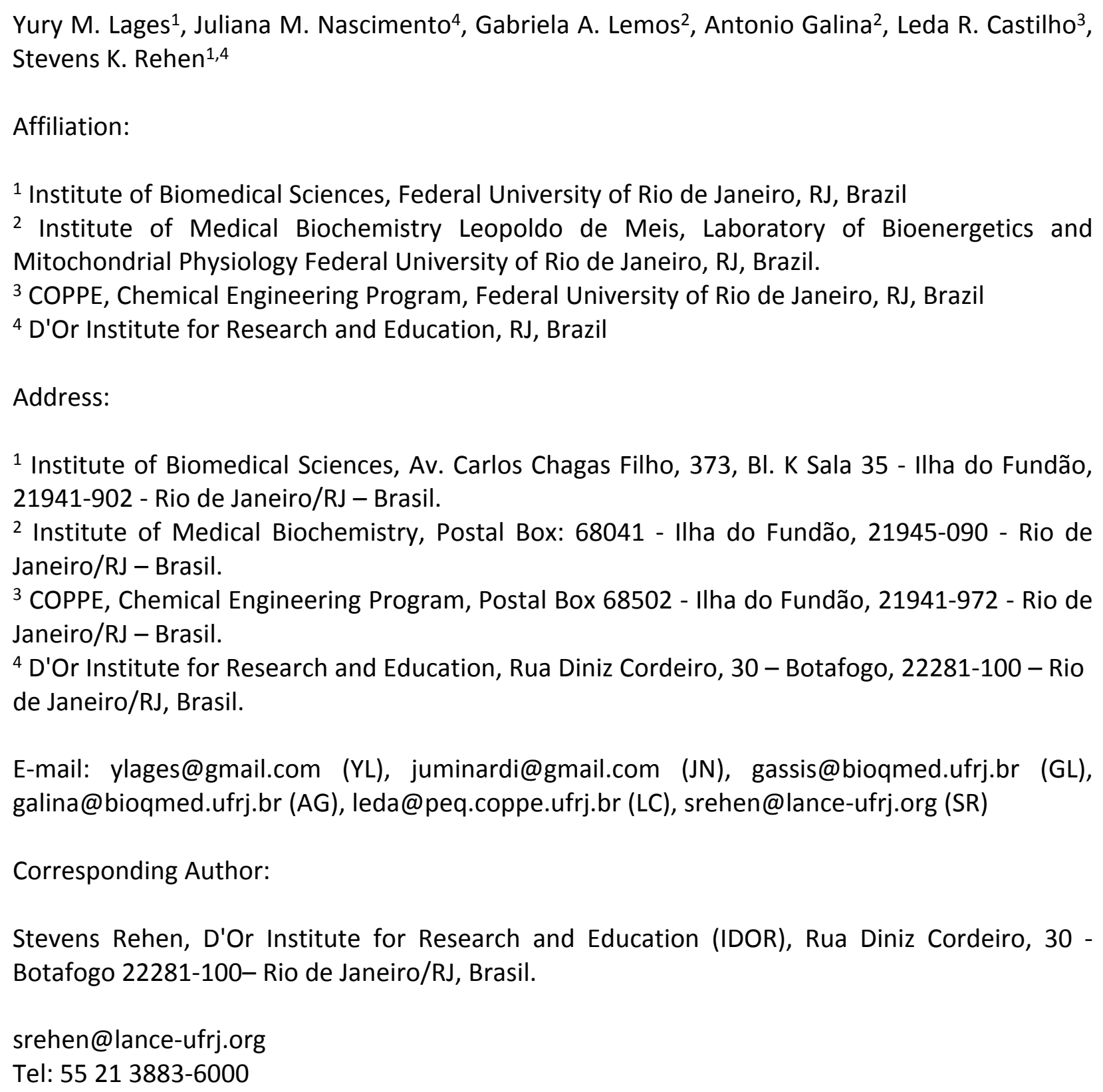




\section{Abstract}

39

40

41

Oxygen concentration should be carefully regulated in all living tissues, beginning at the early embryonic stages. Unbalances in oxygen regulation can lead to cell death and disease. However, to date, few studies have investigated the consequences of variations in oxygen levels for fetal-like cells. Therefore, in the present work, human neural progenitor cells (NPCs) derived from pluripotent stem cells grown in $3 \%$ oxygen $(\mathrm{v} / \mathrm{v})$ were compared with NPCs cultured in $21 \%(\mathrm{v} / \mathrm{v})$ oxygen. Low oxygen concentrations altered the mitochondrial content and oxidative functions of the cells, which led to improved ATP production, while reducing generation of reactive oxygen species (ROS). NPCs cultured in both conditions showed no differences in proliferation and glucose metabolism. Furthermore, antioxidant enzymatic activity was not altered in NPCs cultured in 3\% oxygen under normal conditions, however, when exposed to external agents known to induce oxidative stress, greater susceptibility to DNA damage was observed. Our findings indicate that the management of oxygen levels should be considered for in vitro models of neuronal development and drug screening. 


\section{Introduction}

By describing events that alter mitochondrial metabolism and facilitate tumor formation, Otto Warburg shed light on the importance of oxygen concentrations for cellular health (Warburg 1956). Accordingly, matching the in vitro conditions of a given cell type to the respective in vivo oxygen concentration has become a relevant issue that accompanies the growing number of applications of human pluripotent stem cells, which are particularly relevant for modeling fetal and/or neurological disorders.

Mitochondrial function and oxygen metabolism not only determine aspects of neural development(Li et al. 2004), but they are also strongly implicated in the etiology and progression of brain disorders, including Parkinson's disease, Alzheimer's disease, and Schizophrenia (Paulsen et al. 2013; Yan et al. 2013). Impairment of mitochondrial function or the redox state may be especially problematic for highly metabolically demanding neurons. Mismanagement of these processes is massively problematic, negatively impacting energy metabolism, neurochemical signaling and/or synaptic plasticity, and emergent cognitive processes of these functions (Cheng et al. 2010; Janc \& Muller 2014; Tait \& Green 2012).

Despite the well-recognized relationship between oxidative metabolism and the onset of neural disorders (Paulsen et al. 2013; Yan et al. 2013)' (Carreau et al. 2011), few studies have focused on analyzing changes occurring at atmospheric oxygen concentrations (i.e., $21 \% \mathrm{O}_{2}$ (v/v); typical levels in cell culture, normoxia), compared with physiological levels $\left(3 \% \mathrm{O}_{2}(\mathrm{v} / \mathrm{v})\right)$. Studies carried out on murine neural progenitor cells (NPCs) have considerable differences in proliferation, death, and differentiation (Bae et al. 2012; Chen et al. 2007; Rosafio \& Pellerin 2014; Ross et al. 2012; Stacpoole et al. 2011; Studer et al. 2000). These studies have shown unexpected deviations in cell fate, including altered relative proportions of neuronal and glial populations (Chen et al. 2007; Stacpoole et al. 2011; Studer et al. 2000).

Studies that specifically address the impacts of oxygen levels on the metabolic behavior of NPCs are still rare. Recent reports have described increased dispersion of mitochondria as well as modifications in mitochondrial efficiency and reactive oxygen species (ROS) production 
80 of rat neurons grown under $1-5 \% \mathrm{O}_{2}$ (Tiede et al. 2011). In addition, Tiede and colleagues 81 (Tiede et al. 2011) have reported increased cell death in physiological oxygen concentrations 82 (physioxia (Rosafio \& Pellerin 2014)) when NPCs are exposed to viral infection proteins; 83 however; their study did not elucidate the cause of the alterations. Therefore, the aim of this 84 study was to compare NPCs grown in physioxia and normoxia ( $3 \%$ and $21 \%(\mathrm{v} / \mathrm{v}) \mathrm{O}_{2}$, 85 respectively) in terms of growth kinetics, glycolytic metabolism, mitochondrial content, 86 mitochondrial membrane potential $\left(\Delta \Psi_{M}\right)$, oxygen uptake, ATP production, ROS production, 87 and antioxidant enzymatic activity. 
89

90

91

92

\section{Methods}

\subsection{Differentiation of human embryonic stem cells into NPCS}

To generate NPCs, human embryonic stem cells BR-1 (Fraga et al. 2011) (kindly provided by Prof. Lygia Pereira, São Paulo University - USP) were grown on polystyrene plates (TPP, Switzerland) covered with Matrigel (BD Biosciences, USA) in StemPro medium containing 8 $\mathrm{ng} / \mathrm{mL}$ basic fibroblast growth factor and $0.1 \mathrm{mM} \beta$-mercaptoethanol (all from Thermo Fischer Scientific, USA). After propagation, the cells were differentiated as neural cells using inhibitors of bone morphogenetic protein (Noggin, R\&D Systems, USA), and transforming growth factorbeta (SB431542, Tocris Bioscience, UK) (Chambers et al. 2009). At this moment, cells showed morphology and expression of markers consistent to those of neural progenitor cells (Supplemental information $2 \mathrm{~A}$ and $\mathrm{B}$ ).

\subsection{Cultivation of NPCs in a physiological environment}

NPCs were grown in an environment containing 3\% oxygen (physioxia) in an oxygen control chamber (ProOx model C21, BioSpherix, USA). This equipment was kept at $37^{\circ} \mathrm{C}$ and $5 \%$ $\mathrm{CO}_{2}$, and $3 \% \mathrm{pO}_{2}$ was established by a $\mathrm{N}_{2}$-controlled injection and monitored by an external probe (Mettler Toledo, USA).

\subsection{Growth kinetics}

Cellular growth under different oxygen conditions was evaluated for 18 days ( 3 passages of 6 days). A total of $6 \times 10^{5} \mathrm{NPCs} / \mathrm{mL}$ were plated into 24-well tissue culture plates. Each day, cells from two wells of each condition were detached with Accutase (Millipore, Germany) and counted in a Neubauer chamber.

\subsection{Glucose and lactate measurements}

Glucose and lactate concentrations were determined using a YSI-2700 biochemistry analyzer (Yellow Springs Instruments, USA). This measurement is based on quantification of 
114 hydrogen peroxide generated upon reaction of these organic molecules catalyzed by glucose or 115 lactate oxidases immobilized on membranes.

116

117

118

119

120

121

122

123

124

125

126

127

128

129

130

131

132

133

134

135

136

137

138

139

\subsection{Immunostaining assays}

NPCs were seeded in 96-multiwell $\mu$ Clear dishes (Greiner, Austria) covered with 2.5 $\mu \mathrm{g} / \mathrm{mL}$ laminin (Sigma-Aldrich, USA). After 6 days, these cells were fixed with $4 \%$ paraformaldehyde (Sigma-Aldrich, USA) in phosphate-buffered saline for $30 \mathrm{~min}$.

Then, the cells were treated with 0.5\% Triton X-100 (Sigma-Aldrich, USA), blocked with $5 \%$ bovine serum albumin (Sigma-Aldrich, USA), incubated with the following primary antibodies: rabbit anti-human-histone H2A (H2A.X) (1:100; Cell Signaling, USA), mouse antiNestin (1:100; Chemicon, USA), mouse anti-PSA-NCAM (1:100; Millipore, Germany) and rabbit anti-Tbr2 (1:100; Millipore, Germany). Subsequently, samples were incubated with the following secondary antibodies: goat anti-rabbit AlexaFluor 488 IgG (1:400; Thermo Fischer Scientific, USA) and goat anti-mouse Alexa Fluor 594 IgG (1:400; Thermo Fischer Scientific, USA). Nuclei were stained with $0.5 \mu \mathrm{g} / \mathrm{mL}$ 4'-6-diamino-2-phenylindole (DAPI).

Regions of interest were visualized and identified, and the immunofluorescence emission of the cells was quantified using an Operetta high content analysis system and Harmony software (PerkinElmer, USA). In these experiments, three technical replicates of each biological replicate $(\mathrm{N})$ were performed.

Twenty-five fields per well were chosen randomly. An average of 3.125 nuclei per well were analyzed. The high-content and high-throughput screening platforms used herein allow the evaluation of different samples in a large scale, with simultaneous identification of several parameters using automated fluorescence microscopy, using specific markers for different proteins (Li 2014).

\subsection{Mitochondrial content and $\Delta \Psi_{M}$ quantification assays}

Measurement of the mitochondrial mass of NPCs was performed using $0.3 \mu \mathrm{M}$ Mitotracker DeepRed FM (Thermo Fischer Scientific, USA), a dye that integrates into active 
140 mitochondria (568-nm excitation and 675-nm emission). The $\Delta \Psi_{\mathrm{M}}$ was estimated by cationic 141 staining with 1.6 $\mu \mathrm{M}$ JC-1 (Thermo Fischer Scientific, USA) (488-nm excitation). This dye exists 142 as a monomer at low concentrations, with fluorescence emission at $525 \mathrm{~nm}$ (shown here in 143 green). As it accumulates in the mitochondria, which is membrane potential-dependent, the 144 dye forms aggregates that exhibit a maximum emission at $590 \mathrm{~nm}$ (shown here in yellow). The 145 ratio of aggregate to monomer concentration can be used as a measurement of $\Delta \Psi_{M}$ (Reers et 146 al. 1991).

MitoTracker and JC-1 dyes, diluted in Dulbecco's modified Eagle's medium/F12 (Thermo Fischer Scientific, USA), were applied to NPCs for $40 \mathrm{~min}$ at $37^{\circ} \mathrm{C}$. Fluorescence emission readings were performed in a controlled $5 \% \mathrm{CO}_{2}$ and $37^{\circ} \mathrm{C}$ environment. Hoechst $33342(1 \mu \mathrm{M}$, Thermo Fischer Scientific, USA) was used for nuclear staining.

Thirty-three fields per well were captured randomly. An average of 825 cells were analyzed per well.

\subsection{ROS measurement assay}

Quantification of superoxides was performed using $10 \mu \mathrm{M}$ dihydroethidium (DHE; Thermo Fischer Scientific, USA). This dye, when oxidized in the cytosol, intercalates with DNA and emits fluorescence at $605 \mathrm{~nm}$. DHE was applied similarly to MitoTracker and JC-1. As a positive control, NPCs were induced to produce ROS by a 40 min pretreatment with $3.6 \mu \mathrm{M}$ antimycin A, a mitochondrial complex III inhibitor that stabilizes semi-quinone radicals and favors the escape of electrons to oxygen, thus forming superoxide anions.

Twenty-five fields per well were captured randomly. An average of 6800 nuclei were analyzed per well.

\subsection{Oxygen consumption measurement}

Oxygen consumption was measured by high-resolution respirometry using an Oroboros 
165 acquisition and analysis. NPCs were enzymatically detached from the plate, diluted in culture 166 medium, and seeded to the Oroboros at a concentration of $1 \times 10^{6}$ cells $/ \mathrm{mL}$. The routine 167 oxygen consumption of cells, measured before the addition of modulators of mitochondrial 168 function, was determined after stabilization of the steady state of oxygen consumption for 10 169 15 min. Subsequently, ATP synthesis was inhibited with $2 \mu \mathrm{g} / \mathrm{mL}$ oligomycin. Oxygen consumption related to oxidative phosphorylation coupled to ATP synthesis was determined by the difference between routine respiration and oligomycin-insensitive respiration.

To uncouple oxidative phosphorylation, the protonophore carbonyl cyanide $p$ trifluoromethoxyphenylhydrazone was titrated. The resulting maximum oxygen consumption flux value was established as the maximum capacity of the mitochondrial electron transport system. Finally, the nonoxidative phosphorylation oxygen flux was determined by blocking the electron transporting system with $1 \mu \mathrm{g} / \mathrm{mL}$ antimycin $A$. The residual oxygen flux represents oxidases in the cell sample.

\subsection{Antioxidant enzymatic activity assay}

The activities of the antioxidant enzymes superoxide dismutase (SOD) and glutathione peroxidase (GPx) were measured using commercial kits (Sigma-Aldrich, USA, cat. 19160 and CGP1, respectively). Briefly, the SOD colorimetric assay (440 $\mathrm{nm}$ ) determines the presence of superoxide radicals in a tetrazolium-coupled reaction. The GPx assay measures NADPH depletion (340 nm). The SOD and GPx enzymatic activities were calculated according to the kit instructions. apoptosis during the time of treatment (Supplemental information 1).

\subsection{Statistical analysis}

The unpaired t test was used to compare average differences between two groups. In the case of multiple variable comparisons, one-way analysis of variance was used with the 
191 Bonferroni post-test. The null hypothesis of equality between averages was refuted if $p<0.05$

$192\left(^{*}\right), p<0.01(* *)$, or $p<0.001(* * *)$. Means and standard errors of the mean were plotted. 
193

194

195

196

197

198

199

200

201

202

203

204

205

206

207

208

209

210

211

212

213

214

215

216

217

218

\section{Results}

\subsection{Growth and glycolytic metabolism of human NPCs are not altered in physioxia}

The growth kinetics (Figure 1) as well as glucose uptake and lactate production (Figure 2 ) of NPCs in physioxia $(3 \%, v / v)$ and normoxia $(21 \%, v / v)$ were similar, indicating that impaired cell growth or a metabolic shift from oxidative respiration to glycolysis (Warburg Effect (Warburg 1956)) does not occur in the normoxia-physioxia transition.

\subsection{Mitochondrial content and $\Delta \Psi_{M}$ are altered in NPCs grown in physioxia}

Mitochondrial labeling was performed using the MitoTracker probe, which is internalized by active mitochondria. Fluorescence intensity measurements indicated less mitochondrial content in physioxia-grown NPCs (Figure 3A and B). As a first approach to evaluate mitochondrial function, we quantified $\Delta \Psi_{\mathrm{M}}$ as an indicator of the proton motive force. An increased difference of $\Delta \Psi_{M}$ can be directly related to a strict commitment to ATP formation by FoF $_{1}$ ATP-synthase and/or to decreased proton leakage to the mitochondrial matrix (Jastroch et al. 2010). The data showed increased $\Delta \Psi_{M}$ in physioxia-grown NPCs (Figure $3 C$ and D).

\subsection{Physioxia-grown NPCs feature an increased mitochondrial respiration capacity}

Our data suggest that changes in the mitochondrial physiology are induced by $\mathrm{O}_{2}$ availability (Figure 3). A possible outcome of improved $\Delta \Psi_{\mathrm{M}}$ during mitochondrial function could be a more efficient oxygen consumption directed to ATP production. To verify the full potential of the mitochondria to destine environmental oxygen to oxidative respiration efficiently, we tested the $\mathrm{O}_{2}$ consumption in NPCs grown previously either in normoxia or physioxia in conditions in which plenty of $\mathrm{O}_{2}$ was available, i.e. atmospheric concentrations, by high resolution respirometry. Our data show higher oxygen uptake rates for NPCs previously cultured at $3 \% \mathrm{O}_{2}(\mathrm{v} / \mathrm{v})$. The oxygen flux coupled to ATP production in NPCs cultured at $3 \% \mathrm{O}_{2}$ (v/v) was approximately $70 \%$ higher than that of cells cultured at $20 \% \mathrm{O}_{2}$ (v/v) (Figure 4). Additionally, an increased maximum respiratory capacity of physioxia-grown NPCs was observed, compared to those cultured in normoxia. This observation corroborates our previous 
219

220

221

222

223

224

225

226

227

228

229

230

231

232

233

234

235

236

237

238

239

240

241

242

243

244

results, showing that the NPCs did not suffer from restricted mitochondrial function due to lower oxygen availability. Instead, the physioxia conditions increased the potential of oxidative phosphorylation in NPCs cultured at $3 \% \mathrm{O}_{2}(\mathrm{v} / \mathrm{v})$.

\subsection{NPCs grown in physioxia produce less ROS}

To verify whether increased oxygen consumption could cause physioxia-grown NPCs to produce more ROS, due to impartial oxygen reduction caused by electron leakage from the respiratory chain, we measured ROS production. We used the dye DHE, a permeable probe oxidized in the cytoplasm, which further intercalates DNA and emits fluorescence. As DHE fluorescence was measured in NPCs in their native oxygen environments (i.e., normoxia and physioxia), we observed that, under routine conditions, ROS production was equivalent in both situations (Figure 5). However, after antimycin A addition, physioxia-grown cells showed lower ROS levels from mitochondrial complex III induced by semi-quinone radical stabilization, compared to normoxia conditions (Figure 5).

These results suggest that NPCs grown under physiological oxygen are more resistant to redox imbalance. Moreover, this finding also suggests that their antioxidant defense system may be more efficient to scavenge the mitochondrial ROS generated.

\subsection{GPx activity is lower in NPCs grown in physioxia}

To assess whether the antioxidant defense system of physioxia-grown NPCs is more active under routine conditions, we measured the activities of SOD and GPx in these cells. SOD is responsible for catalyzing the dismutation of superoxide anions in hydrogen peroxide and water. Then, GPx oxidizes intracellular glutathione, reducing peroxide to alcohol and water.

Our data show that NPCs grown either in normoxia or physioxia have equivalent enzymatic activities; however, when treated with antimycin A, the NPCs cultured under lower oxygen concentrations showed a less-pronounced increase of GPx activity in response to the ROS increase, while the SOD activity level was sustained (Figure 6). This finding indicates that, unlike what was hypothesized previously, NPCs grown in physioxia and treated with antimycin 
245 A do not show decreased ROS production due to greater antioxidant activity in the pathway 246 analyzed herein. Instead, GPx, one of the core enzymes of the cellular antioxidant machinery, 247 depicts a decreased response to the ROS increase in these NPCs.

Although the glutathione pathway is not the only one involved in superoxide anion detoxification, it seems to be extremely relevant in NPCs, even surpassing the activity of catalase and peroxiredoxin 3, as shown previously (Mitozo et al. 2011; Xi et al. 2014). Taken together, these data suggest that, in fact, the antioxidant defense enzymes may be downregulated in physiological oxygen concentrations, compared to atmospheric ones.

\subsection{Physioxia-grown NPCs suffer increased DNA damage when exposed to hydrogen} peroxide

Although physioxia-grown NPCs produced lower levels of ROS when treated with antimycin A (Figure 5), our data show that these cells also had a decreased activity of GPx (Figure 6), one of the main enzymes responsible for peroxide detoxification. Therefore, we investigated whether NPCs cultivated under low oxygen concentrations would be more susceptible to stress. To this end, we quantified DNA damage, an indication of oxidative stress (Konyalioglu et al. 2013), in NPCs grown under routine conditions and after hydrogen peroxide treatment.

To quantify the impact of these treatments on DNA damage, the antibody against H2A.X was used to measure altered DNA. H2A.X is phosphorylated when DNA strands break, which signals for DNA repair and cell cycle arrest. Staining of H2A.X appears within the nucleus and can be monitored by both the overall fluorescence intensity and the number of visibly detectable aggregated structures.

Under our routine conditions, we observed that NPCs grown in normoxia or physioxia had equivalent levels of DNA damage (Figure 7). However, after exposure to hydrogen peroxide, cells cultured under lower oxygen concentrations showed increased DNA damage, which was observed by increased fluorescence intensity both in the whole nucleus and in H2A.X spots (Figure 7C). Moreover, the population of nuclei containing more than 20 spots decreased; 
272 while the whole stained nuclei population, which depicted so many spots that they became 273 indistinguishable from one another, was increased in physioxia-grown NPCs (Figure 7D).

274 Increased H2A.X staining of NPCs grown in physioxia and treated with hydrogen 275 peroxide suggests that these cells are more susceptible to exogenous ROS than those cultured 276 in normoxia. Taken together, our results show that higher susceptibility to oxidative stress 277 could be partly due to a lower response of antioxidant enzymes to stress agents in physioxia278 grown NPCs, which could contribute to decreased resistance to oxidative stress. 


\section{Discussion}

Evidence of increased susceptibility to exogenous stress agents in physioxia-grown NPCs highlights the importance of culturing human neural cells under low oxygen conditions to better evaluate the effect of drugs, especially the ones known to trigger oxidative stress.

We observed that NPCs in physioxia had reduced mitochondrial content, without changes in morphology (Supplemental information 2A), which might indicate stress (Giedt et al. 2012). Other studies have described lower mitochondrial DNA mass in human carcinoma, which is known to have a decreased oxygen gradient from the border to the center (Chiba et al. 2013). Moreover, the NPCs showed no alterations in fusion or fission of the mitochondria, which are linked to the dynamics of these organelles and influence their morphology and amount (Chan 2012).

Although reductions in mitochondrial content can lead to a decrease in ATP produced by oxidative phosphorylation (Clanton et al. 2013), the lack of bioenergetics compensation by increased glycolysis and an unaltered growth profile of physioxia-grown NPCs do not indicate reduced mitochondrial activity in these cells. Thus, we evaluated the hypothesis that, instead, there may be improved function of these organelles to maintain ATP production rates necessary for cellular metabolism, even under low $\mathrm{O}_{2}$ concentrations.

Our data reveal that the mitochondria increased the $\triangle \Psi_{M}$ in physioxia-grown NPCs, indicating a higher activity and mitochondrial efficiency in ATP synthesis. As previous reports have demonstrated that mitochondrial membrane potential increases as oxygen availability is lowered below certain levels (Tiede et al. 2011), we believe our system is beneath this threshold. To confirm our hypothesis that increased $\Delta \Psi_{M}$ could directly affect mitochondrial efficiency and commitment to oxidative respiration in physioxia, we investigated the parameters related to ATP production by measuring the oxygen consumption rates in an atmospheric environment, where oxygen is abundant.

Our high-resolution respirometry results corroborate that, in physioxia, oxygen consumption used for ATP production is increased. Moreover, using this technique, we 
306 concluded that a greater $\Delta \Psi_{\mathrm{M}}$ could allow cells to maintain ATP production by oxidative 307 phosphorylation with fewer mitochondria. Thus, we hypothesized that this outcome may 308 reflect an adaptive mechanism conferring a more efficient use of scarce oxygen.

Notably, even with the increased potential respiratory capacity, ROS production was not altered in physioxia compared to normoxia. Notwithstanding, when NPCs were exposed to antimycin A, stimulating an enhanced ROS production by mitochondria (Chen et al. 2003), we observed a greater amount of ROS in normoxia-grown NPCs. In this oxygen environment, oxygen availability as a source of ROS production is much higher. Thus, when electron leakage from the respiratory chain is stimulated by antimycin $A$ and the oxygen concentrations increase, superior ROS formation is expected. On the other hand, in physioxia, conditions in which the free oxygen concentration is reduced, the ROS production is decreased even when NPCs are treated with antimycin A, corroborating our hypothesis of greater mitochondrial commitment to ATP production.

Keeping in mind the core importance of the antioxidant enzymes SOD and GPx in the detoxification process of superoxide anions generated by mitochondria in neural cells (even overcoming catalase activity (Mitozo et al. 2011)), we evaluated whether the decreased production of these ROS was a consequence not only of increased $\Delta \Psi_{M}$ but also of improved enzymatic activity.

The SOD activity measurements revealed that this enzyme function was not altered in physioxia, neither under routine conditions nor after antimycin A treatment (Figure 6). As superoxide anion dismutation in hydrogen peroxide can also occur spontaneously, ROS production can increase after antimycin A treatment even if there are no alterations in SOD activity (Drose \& Brandt 2008).

GPx activity was also shown to be very similar between NPCs grown in normoxia or physioxia in routine conditions. However, after antimycin A treatment, cells grown in low oxygen concentrations showed a decreased response, while those cultured in a standard oxygen environment showed a greater increase in GPx activity. While no consensus currently 
333

334

335

336

337

338

339

340

341

342

343

exists, earlier studies correlating reduced oxygen concentrations to GPx activity indicate decreased activity of this and other enzymes involved in ROS detoxification, such as peroxiredoxin 3 (Becker et al. 2014; Duranton et al. 2012; Hidalgo et al. 2014; Xi et al. 2014). Indeed, minor increases of ROS production in response to antimycin A treatment were not enough to generate greater GPx activation in physioxia-grown NPCs.

The importance of GPx peroxide detoxification was investigated once more, measuring the susceptibility of these cells to hydrogen peroxide insults by quantifying the amount of DNA damage, a marker of oxidative stress. Increased DNA damage caused by peroxide, shown previously in other models (Konyalioglu et al. 2013; Mitozo et al. 2011), was herein reproduced by NPCs in normoxia. These cells showed a greater H2A.X fluorescence intensity and more spots in the nuclei, and the amount of marked cells rose from $25 \%$ to $100 \%$ after treatment.

Comparing NPCs grown in normoxia vs. physioxia, all parameters indicated a similar amount of DNA damage in routine conditions (without insult). However, after hydrogen peroxide insult, physioxia-grown NPCs showed nuclei with increased H2A.X fluorescence and more spots, indicating increased DNA damage as compared to normoxia-grown NPCs.

These data indicate a greater susceptibility of human NPCs grown in physiological oxygen conditions to insults caused by ROS. This evidence is of great relevance, since models to study disease related to oxidative stress are usually performed under atmospheric oxygen environments, and possibly may not represent actual processes and responses.

Recent studies, using human NPCs differentiated from donors with brain disorders, have shown alterations in mitochondrial membrane potential and oxidative stress, in addition to increased DNA damage, when compared to control NPCs generated from healthy donors; these data indicate phenotype reversibility when classical drugs are used (Brennand et al. 2014; Paulsen et al. 2013). Just as these studies, many others have been performed under atmospheric oxygen concentrations; thus, the results could be more reliable if the cells had been grown at the same oxygen concentrations found in vivo. 
Although there are only suggestions of how cell cultivation under physiological oxygen 360 levels could alter disease modeling in vitro, cell and metabolic modifications induced by low 361 oxygen environments do occur. Tumor and mesodermal adult stem cell studies have elucidated 362 several mechanisms on how physioxia would alter activation of cellular pathways (Eliasson \& 363 Jonsson 2010; Erler et al. 2004; Forristal et al. 2013b; Jang \& Sharkis 2007; Kubota et al. 2008; 364 Rosova et al. 2008; Song et al. 2006), such as the hypoxia-inducible factor 1 alpha mechanism. 365 On the other hand, studies with pluripotent stem cells (Forristal et al. 2013a; Narva et al. 2013; 366 Pereira et al. 2013; Zachar et al. 2010) and derived NPCs have focused on describing how cell 367 differentiation would be affected by oxygen concentrations. In this study, we demonstrated 368 that alterations in physioxia-grown NPCs at the metabolic level not only occur but also lead to a 369 differential response to exogenous oxidative agents (Figure 8), thus shedding light on the 370 importance of oxygenation control in studies focusing on disease and drug screening assays, in 371 a scalable, efficient, and unbiased manner.

\section{Acknowledgements}

374 This work is part of the MSc thesis of YL. We thank Michelle Kormann for the support during cell 375 cultivation. 


\section{References}

Bae D, Mondragon-Teran P, Hernandez D, Ruban L, Mason C, Bhattacharya SS, and Veraitch FS. 2012. Hypoxia enhances the generation of retinal progenitor cells from human induced pluripotent and embryonic stem cells. Stem Cells Dev 21:1344-1355. 10.1089/scd.2011.0225

Becker NP, Martitz J, Renko K, Stoedter M, Hybsier S, Cramer T, and Schomburg L. 2014. Hypoxia reduces and redirects selenoprotein biosynthesis. Metallomics 6:1079-1086. $10.1039 / \mathrm{c} 4 \mathrm{mt} 00004 \mathrm{~h}$

Brennand K, Savas JN, Kim Y, Tran N, Simone A, Hashimoto-Torii K, Beaumont KG, Kim HJ, Topol A, Ladran I, Abdelrahim M, Matikainen-Ankney B, Chao SH, Mrksich M, Rakic P, Fang G, Zhang B, Yates JR, 3rd, and Gage FH. 2014. Phenotypic differences in hiPSC NPCs derived from patients with schizophrenia. Mol Psychiatry. mp201422 [pii]

10.1038/mp.2014.22

Carreau A, El Hafny-Rahbi B, Matejuk A, Grillon C, and Kieda C. 2011. Why is the partial oxygen pressure of human tissues a crucial parameter? Small molecules and hypoxia. J Cell Mol Med 15:12391253. 10.1111/j.1582-4934.2011.01258.x

Chambers SM, Fasano CA, Papapetrou EP, Tomishima M, Sadelain M, and Studer L. 2009. Highly efficient neural conversion of human ES and iPS cells by dual inhibition of SMAD signaling. Nat Biotechnol 27:275-280. nbt.1529 [pii]

$10.1038 /$ nbt.1529

Chan DC. 2012. Fusion and fission: interlinked processes critical for mitochondrial health. Annu Rev Genet 46:265-287. 10.1146/annurev-genet-110410-132529

Chen HL, Pistollato F, Hoeppner DJ, Ni HT, McKay RD, and Panchision DM. 2007. Oxygen tension regulates survival and fate of mouse central nervous system precursors at multiple levels. Stem Cells 25:2291-2301. 2006-0609 [pii]

10.1634/stemcells.2006-0609

Chen Q, Vazquez EJ, Moghaddas S, Hoppel CL, and Lesnefsky EJ. 2003. Production of reactive oxygen species by mitochondria: central role of complex III. J Biol Chem 278:36027-36031. 10.1074/jbc.M304854200

M304854200 [pii]

Cheng A, Hou Y, and Mattson MP. 2010. Mitochondria and neuroplasticity. ASN Neuro 2:e00045. 10.1042/AN20100019

Chiba M, Yokoyama C, Okada M, and Hisatomi H. 2013. Mitochondrial DNA reduced by hypoxic conditions in three-dimensional (3D) spheroid cell cultures. Tumour Biol. 10.1007/s13277-0142593-6

Clanton TL, Hogan MC, and Gladden LB. 2013. Regulation of cellular gas exchange, oxygen sensing, and metabolic control. Compr Physiol 3:1135-1190. 10.1002/cphy.c120030

Drose S, and Brandt U. 2008. The mechanism of mitochondrial superoxide production by the cytochrome bc1 complex. J Biol Chem 283:21649-21654. M803236200 [pii]

10.1074/jbc.M803236200

Duranton C, Rubera I, Cougnon M, Melis N, Chargui A, Mograbi B, and Tauc M. 2012. CFTR is involved in the fine tuning of intracellular redox status: physiological implications in cystic fibrosis. $A m ~ J$ Pathol 181:1367-1377. S0002-9440(12)00492-0 [pii]

10.1016/j.ajpath.2012.06.017

Eliasson P, and Jonsson JI. 2010. The hematopoietic stem cell niche: low in oxygen but a nice place to be. J Cell Physiol 222:17-22. 10.1002/jcp.21908

Erler JT, Cawthorne CJ, Williams KJ, Koritzinsky M, Wouters BG, Wilson C, Miller C, Demonacos C, Stratford IJ, and Dive C. 2004. Hypoxia-mediated down-regulation of Bid and Bax in tumors 
occurs via hypoxia-inducible factor 1-dependent and -independent mechanisms and contributes to drug resistance. Mol Cell Biol 24:2875-2889.

Forristal CE, Christensen DR, Chinnery FE, Petruzzelli R, Parry KL, Sanchez-Elsner T, and Houghton FD. 2013a. Environmental oxygen tension regulates the energy metabolism and self-renewal of human embryonic stem cells. PLoS One 8:e62507. 10.1371/journal.pone.0062507

PONE-D-11-21077 [pii]

Forristal CE, Winkler IG, Nowlan B, Barbier V, Walkinshaw G, and Levesque JP. 2013b. Pharmacologic stabilization of HIF-1alpha increases hematopoietic stem cell quiescence in vivo and accelerates blood recovery after severe irradiation. Blood 121:759-769. blood-2012-02-408419 [pii]

10.1182/blood-2012-02-408419

Fraga AM, Sukoyan M, Rajan P, Braga DP, laconelli A, Jr., Franco JG, Jr., Borges E, Jr., and Pereira LV. 2011. Establishment of a Brazilian line of human embryonic stem cells in defined medium: implications for cell therapy in an ethnically diverse population. Cell Transplant 20:431-440. ct0028fraga [pii]

10.3727/096368910X522261

Giedt RJ, Pfeiffer DR, Matzavinos A, Kao CY, and Alevriadou BR. 2012. Mitochondrial dynamics and motility inside living vascular endothelial cells: role of bioenergetics. Ann Biomed Eng 40:19031916. 10.1007/s10439-012-0568-6

Hidalgo M, Marchant D, Quidu P, Youcef-Ali K, Richalet JP, Beaudry M, Besse S, and Launay T. 2014. Oxygen Modulates the Glutathione Peroxidase Activity during the L6 Myoblast Early Differentiation Process. Cell Physiol Biochem 33:67-77. 000356650 [pii]

10.1159/000356650

Janc OA, and Muller M. 2014. The free radical scavenger Trolox dampens neuronal hyperexcitability, reinstates synaptic plasticity, and improves hypoxia tolerance in a mouse model of Rett syndrome. Front Cell Neurosci 8:56. 10.3389/fncel.2014.00056

Jang YY, and Sharkis SJ. 2007. A low level of reactive oxygen species selects for primitive hematopoietic stem cells that may reside in the low-oxygenic niche. Blood 110:3056-3063. blood-2007-05087759 [pii]

10.1182/blood-2007-05-087759

Jastroch M, Divakaruni AS, Mookerjee S, Treberg JR, and Brand MD. 2010. Mitochondrial proton and electron leaks. Essays Biochem 47:53-67. bse0470053 [pii]

10.1042/bse0470053

Konyalioglu S, Armagan G, Yalcin A, Atalayin C, and Dagci T. 2013. Effects of resveratrol on hydrogen peroxide-induced oxidative stress in embryonic neural stem cells. Neural Regen Res 8:485-495. 10.3969/j.issn.1673-5374.2013.06.001

NRR-8-485 [pii]

Kubota Y, Takubo K, and Suda T. 2008. Bone marrow long label-retaining cells reside in the sinusoidal hypoxic niche. Biochem Biophys Res Commun 366:335-339. S0006-291X(07)02480-1 [pii]

10.1016/j.bbrc.2007.11.086

Li Z. 2014. Editorial: high content screening for lead identification and optimization. Curr Chem Genomics Transl Med 8:1-2. 10.2174/2213988501408010001

CCGTM-8-1 [pii]

Li Z, Okamoto K, Hayashi Y, and Sheng M. 2004. The importance of dendritic mitochondria in the morphogenesis and plasticity of spines and synapses. Cell 119:873-887. S0092867404010451 [pii]

10.1016/j.cell.2004.11.003

Mitozo PA, de Souza LF, Loch-Neckel G, Flesch S, Maris AF, Figueiredo CP, Dos Santos AR, Farina M, and Dafre AL. 2011. A study of the relative importance of the peroxiredoxin-, catalase-, and 
glutathione-dependent systems in neural peroxide metabolism. Free Radic Biol Med 51:69-77. S0891-5849(11)00174-2 [pii]

10.1016/j.freeradbiomed.2011.03.017

Narva E, Pursiheimo JP, Laiho A, Rahkonen N, Emani MR, Viitala M, Laurila K, Sahla R, Lund R, Lahdesmaki H, Jaakkola $P$, and Lahesmaa R. 2013. Continuous Hypoxic Culturing of Human Embryonic Stem Cells Enhances SSEA-3 and MYC Levels. PLOS One 8:e78847. 10.1371/journal.pone.0078847

PONE-D-13-14987 [pii]

Paulsen S, da Silveira MS, Galina A, and Rehen SK. 2013. Pluripotent stem cells as a model to study oxygen metabolism in neurogenesis and neurodevelopmental disorders. Arch Biochem Biophys 534:3-10. S0003-9861(12)00377-3 [pii]

\subsection{6/j.abb.2012.10.009}

Pereira SL, Graos M, Rodrigues AS, Anjo SI, Carvalho RA, Oliveira PJ, Arenas E, and Ramalho-Santos J. 2013. Inhibition of Mitochondrial Complex III Blocks Neuronal Differentiation and Maintains Embryonic Stem Cell Pluripotency. PLoS One 8:e82095. 10.1371/journal.pone.0082095

PONE-D-13-31266 [pii]

Reers M, Smith TW, and Chen LB. 1991. J-aggregate formation of a carbocyanine as a quantitative fluorescent indicator of membrane potential. Biochemistry 30:4480-4486.

Rosafio K, and Pellerin L. 2014. Oxygen tension controls the expression of the monocarboxylate transporter MCT4 in cultured mouse cortical astrocytes via a hypoxia-inducible factor-1alphamediated transcriptional regulation. Glia 62:477-490. 10.1002/glia.22618

Rosova I, Dao M, Capoccia B, Link D, and Nolta JA. 2008. Hypoxic preconditioning results in increased motility and improved therapeutic potential of human mesenchymal stem cells. Stem Cells 26:2173-2182. 2007-1104 [pii]

10.1634/stemcells.2007-1104

Ross HH, Sandhu MS, Cheung TF, Fitzpatrick GM, Sher WJ, Tiemeier AJ, Laywell ED, and Fuller DD. 2012. In vivo intermittent hypoxia elicits enhanced expansion and neuronal differentiation in cultured neural progenitors. Exp Neurol 235:238-245. S0014-4886(12)00059-3 [pii]

10.1016/j.expneurol.2012.01.027

Song X, Liu X, Chi W, Liu Y, Wei L, Wang X, and Yu J. 2006. Hypoxia-induced resistance to cisplatin and doxorubicin in non-small cell lung cancer is inhibited by silencing of HIF-1alpha gene. Cancer Chemother Pharmacol 58:776-784. 10.1007/s00280-006-0224-7

Stacpoole SR, Bilican B, Webber DJ, Luzhynskaya A, He XL, Compston A, Karadottir R, Franklin RJ, and Chandran S. 2011. Derivation of neural precursor cells from human ES cells at $3 \% O(2)$ is efficient, enhances survival and presents no barrier to regional specification and functional differentiation. Cell Death Differ 18:1016-1023. cdd2010171 [pii]

10.1038/cdd.2010.171

Studer L, Csete M, Lee SH, Kabbani N, Walikonis J, Wold B, and McKay R. 2000. Enhanced proliferation, survival, and dopaminergic differentiation of CNS precursors in lowered oxygen. I Neurosci 20:7377-7383.

Tait SW, and Green DR. 2012. Mitochondria and cell signalling. J Cell Sci 125:807-815. 125/4/807 [pii] 10.1242/jcs.099234

Tiede LM, Cook EA, Morsey B, and Fox HS. 2011. Oxygen matters: tissue culture oxygen levels affect mitochondrial function and structure as well as responses to HIV viroproteins. Cell Death Dis 2:e246. cddis2011128 [pii]

10.1038/cddis.2011.128

Warburg O. 1956. On the origin of cancer cells. Science 123:309-314. 
518 Xi H, Gao YH, Han DY, Li QY, Feng LJ, Zhang W, Ji G, Xiao JC, Zhang HZ, and Wei Q. 2014. Hypoxia inducible factor-1alpha suppresses Peroxiredoxin 3 expression to promote proliferation of CCRCC cells. FEBS Lett 588:3390-3394. S0014-5793(14)00579-1 [pii]

10.1016/j.febslet.2014.07.030

Yan MH, Wang X, and Zhu X. 2013. Mitochondrial defects and oxidative stress in Alzheimer disease and Parkinson disease. Free Radic Biol Med 62:90-101. S0891-5849(12)01823-0 [pii]

10.1016/j.freeradbiomed.2012.11.014

Zachar V, Prasad SM, Weli SC, Gabrielsen A, Petersen K, Petersen MB, and Fink T. 2010. The effect of human embryonic stem cells (hESCs) long-term normoxic and hypoxic cultures on the maintenance of pluripotency. In Vitro Cell Dev Biol Anim 46:276-283. 10.1007/s11626-010-93053

530 
531

532

533

534

535

536

537

538

539

540

541

542

543

544

545

546

547

548

549

550

551

552

553

554

555

556

557

558

559

560

561

562

\section{FIGURE LEGENDS}

Figure 1: Comparison of the growth profiles of NPCs cultured either in physioxia (gray line) or normoxia (black line) over an 18-day period, including passaging on days 6 and 12.

Figure 2: Glucose uptake and lactate concentration in the supernatant of NPCs grown in physioxia (gray lines) and normoxia (black lines) throughout the first, second, and third passages.

Figure 3: Evaluation of mitochondrial content by MitoTracker ( $A$ and $B$ ), in red; and mitochondrial membrane potential by JC-1 (C and D), in green and yellow. Nuclei are stained in blue by DAPI. $p<0.05\left(^{*}\right), p<0.01\left({ }^{* *}\right)$. Scale bar: $50 \mu \mathrm{m}$.

Figure 4: Oxygen uptake quantification of NPCs grown either in physioxia (gray bars) or normoxia (black bars) by high-resolution respirometry, providing the following parameters: routine respiration, oxygen consumption dedicated to ATP synthesis, maximum mitochondrial capacity, and extra-mitochondrial respiration. $\mathrm{p}<0.05\left(^{*}\right), \mathrm{p}<0.001\left(^{* * *}\right)$.

Figure 5: ROS quantification with the probe DHE. $p<0.01\left(^{* *}\right)$.

Figure 6: Enzymatic activity of the antioxidant enzymes superoxide dismutase (SOD) and glutathione peroxidase (GPX). Activity quantification under routine conditions and after antimycin A treatment, which stimulates maximum ROS production. $p<0.05\left(^{*}\right)$.

Figure 7: DNA damage as evidenced by H2A.X (green), either after hydrogen peroxide treatment $(A)$ or under routine conditions (B). Total nuclei are stained with DAPI (blue). Whole nucleus fluorescence is shown in both oxygen concentrations, after hydrogen peroxide treatment (C) and under routine conditions (D). The fluorescence of the whole nucleus and of the H2A.X spots was analyzed in the four categories. $p<0.01\left(^{* *}\right), p<0.001\left(^{* * *}\right)$. Scale bar: 25 $\mu \mathrm{m}$.

Figure 8: Graphic summary of the results 


\section{CAPTION FOR SUPPLEMENTARY FIGURE:}

\section{Supplementary Figure 1}

565 NPCs grown either in physiological oxygen concentrations (gray bars), or in normoxia (black 566 bars), do not show differences in apoptosis after treated with $3.6 \mu \mathrm{M}$ antimycin A for $40 \mathrm{~min}$.

\section{Supplementary Figure 2}

568 NPCs grown in physiological oxygen concentrations show the same morphology, and expression 569 of characteristic markers, as those propagated in normoxia. Bright field and 570 immunofluorescence images are displayed in (A), and quantification of classic NPC markers, 571 such as PSA-NCAM, Nestin and Tbr2 are shown as for total amount of stained cells in (B), and as 572 their fluorescence intensity in (C). Scale bars: $200 \mu \mathrm{m}$ for bright field images, $100 \mu \mathrm{m}$ for PSA573 NCAM and Nestin, and $50 \mu \mathrm{m}$ for Tbr2.

574 
575 Figure 1.

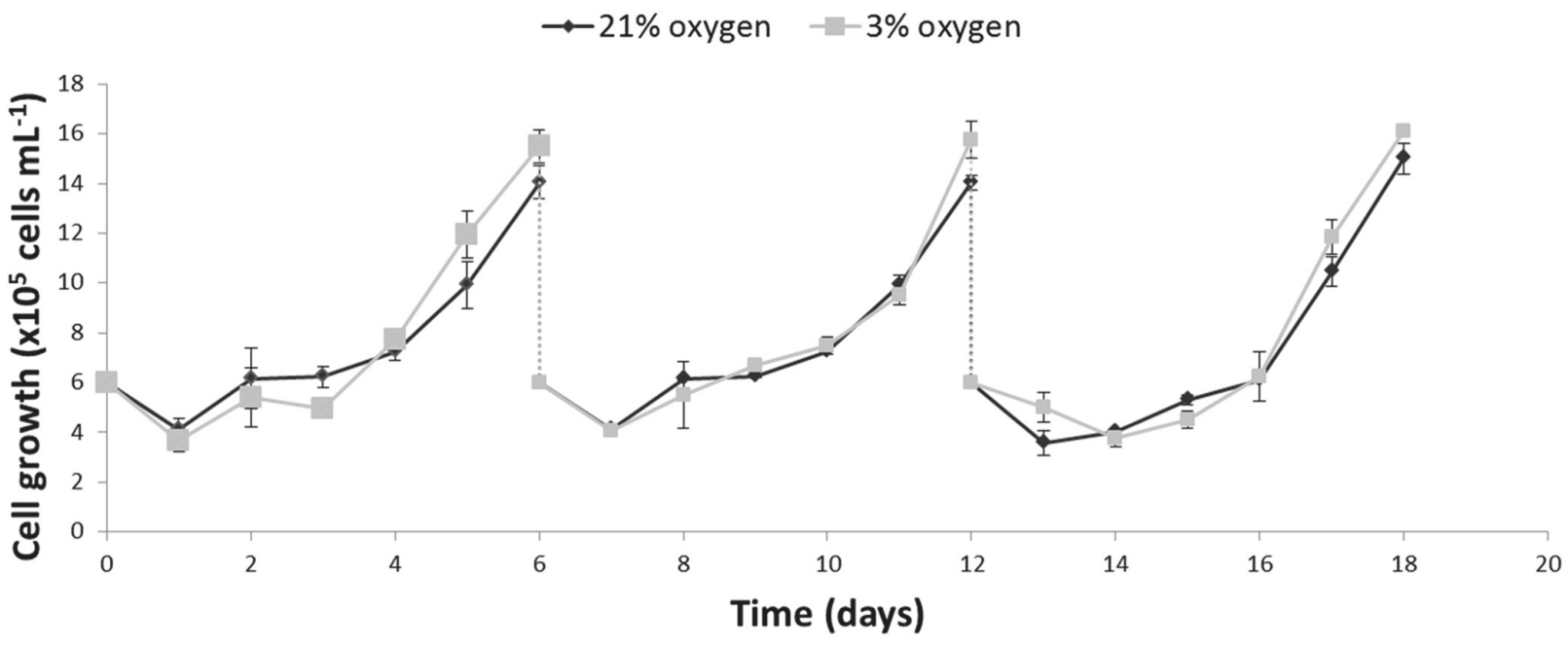




\section{Figure 2.}

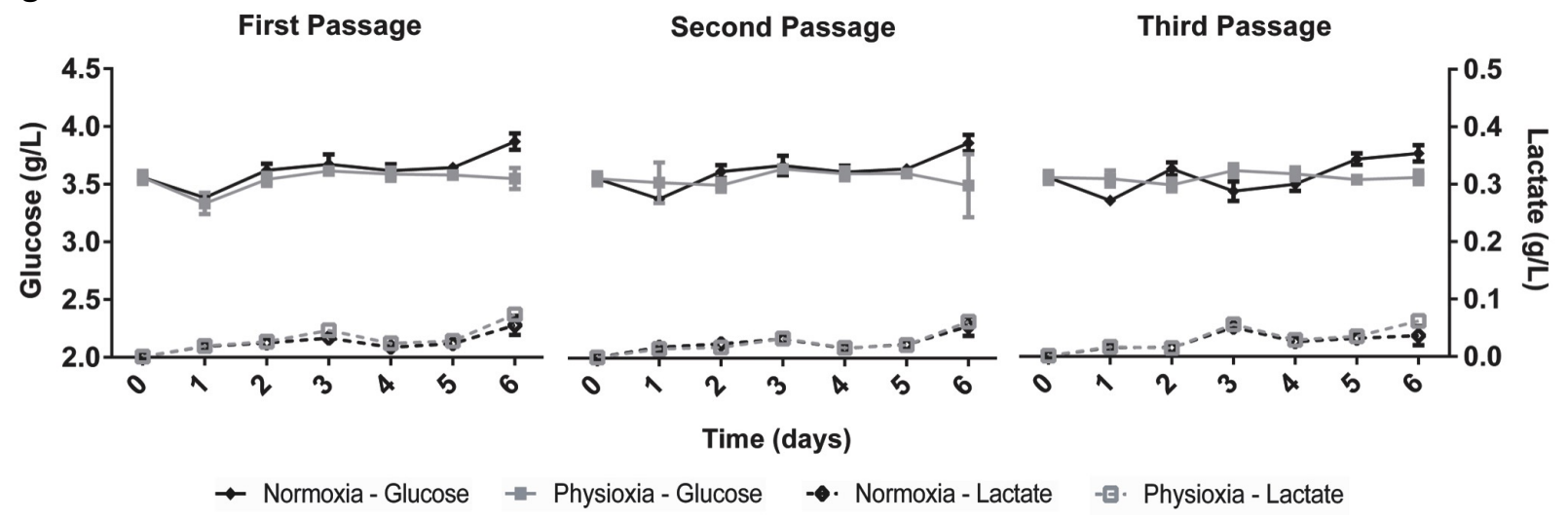


579 Figure 3.
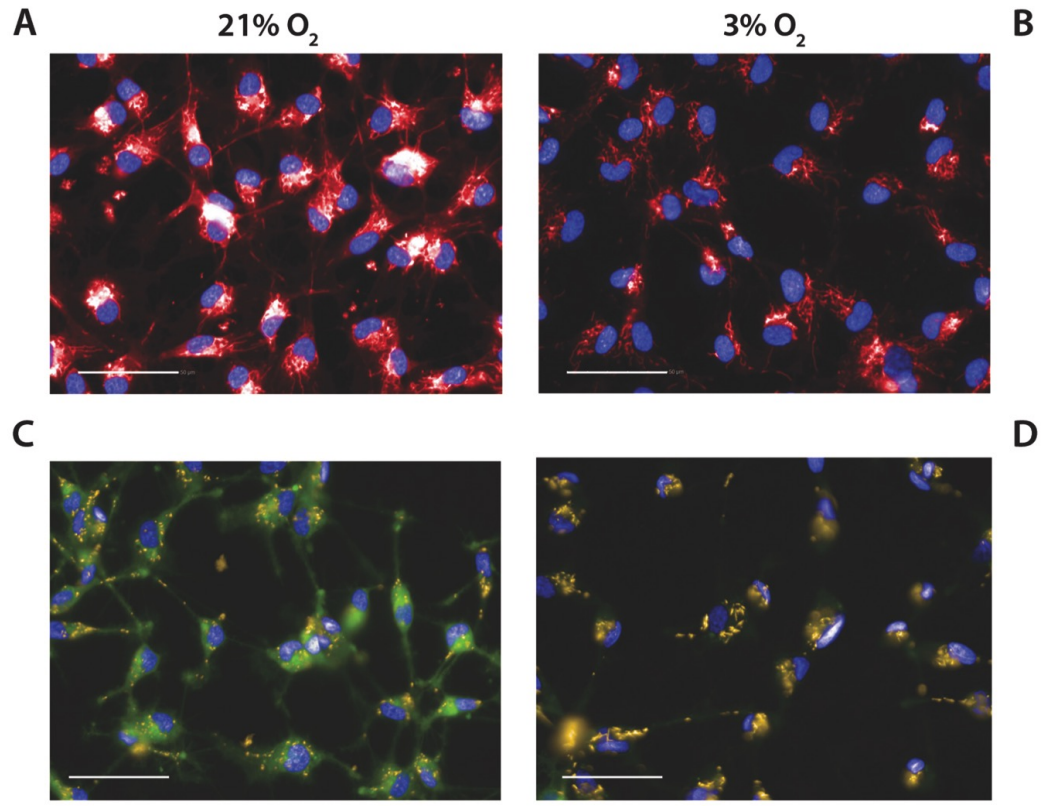

B

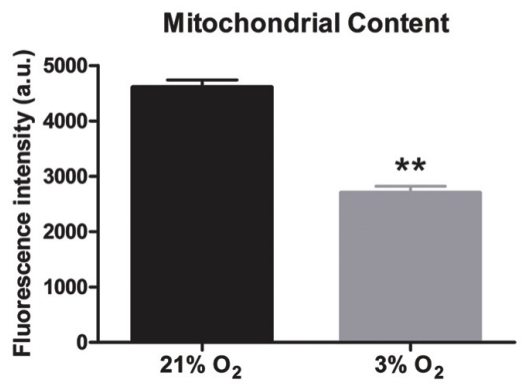

D

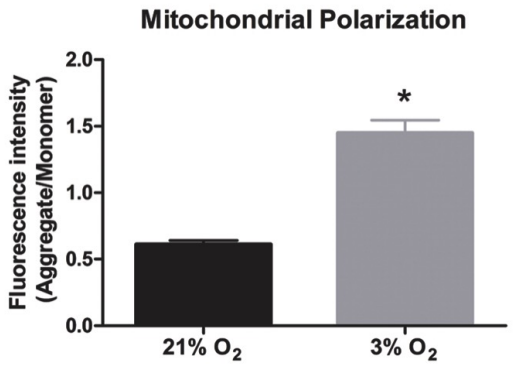


$581 \quad$ Figure 4.

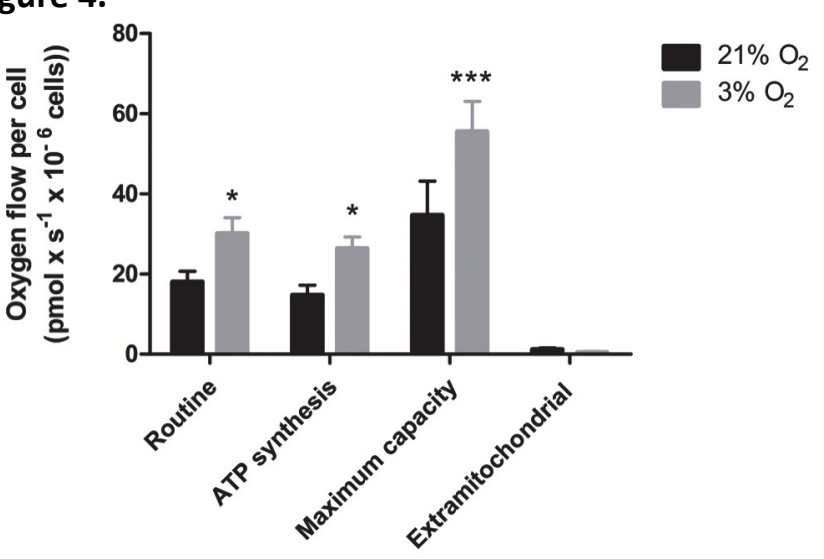


$583 \quad$ Figure 5.

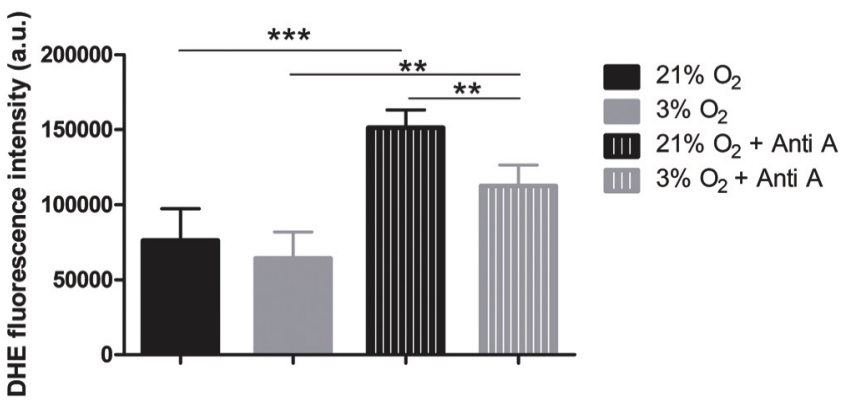


585 Figure 6.
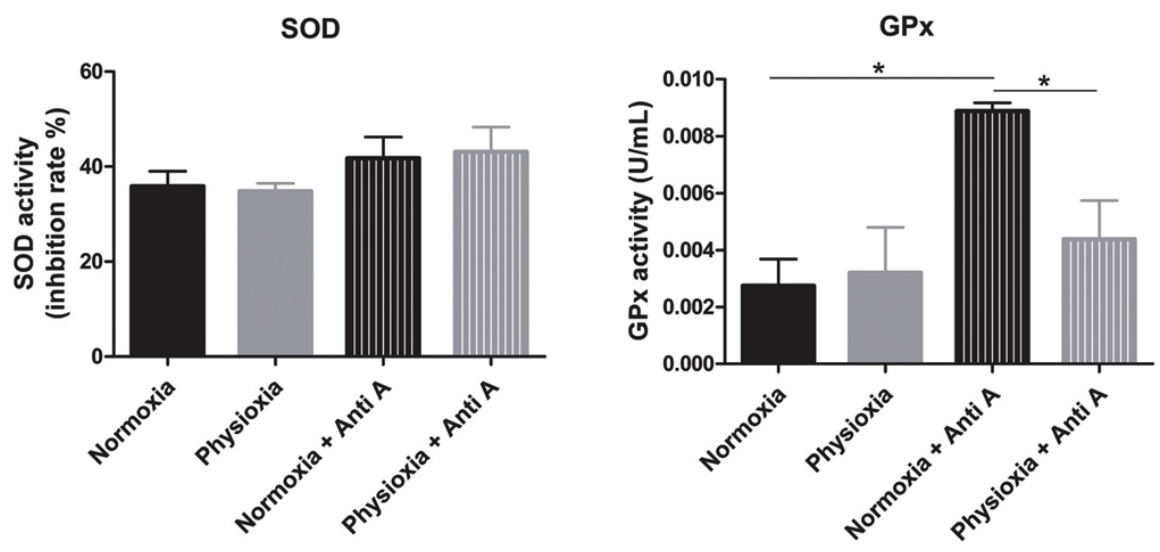

$21 \% \mathrm{O}_{2}$

$3 \% \mathrm{O}_{2}$

III $21 \% \mathrm{O}_{2}+$ Anti $\mathrm{A}$

III $3 \% \mathrm{O}_{2}+$ Anti $\mathrm{A}$

586 


\section{Figure 7.}

A

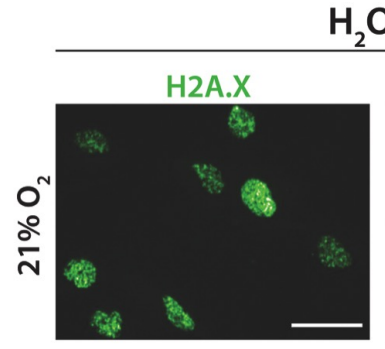

$\mathrm{H}_{2} \mathrm{O}_{2}$
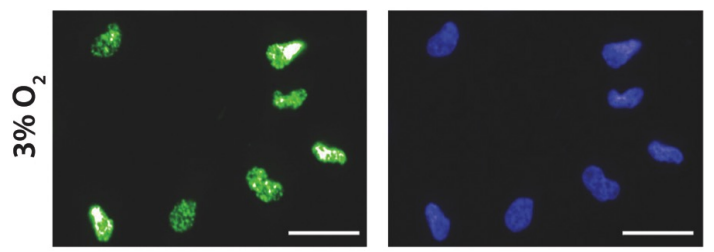

C

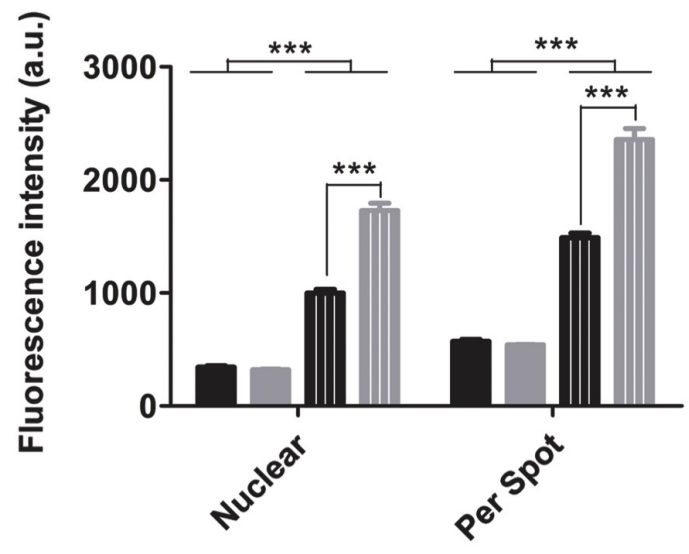

$21 \% \mathrm{O}_{2}$

$3 \% \mathrm{O}_{2}$

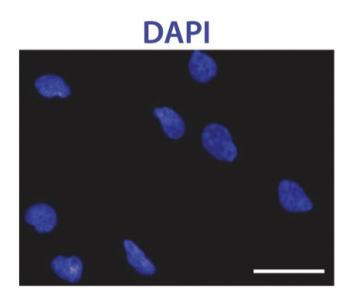

588
B

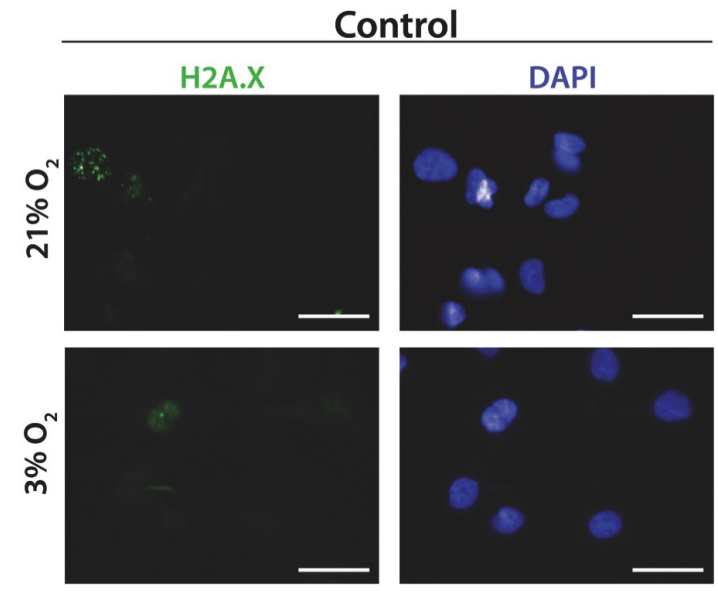

D

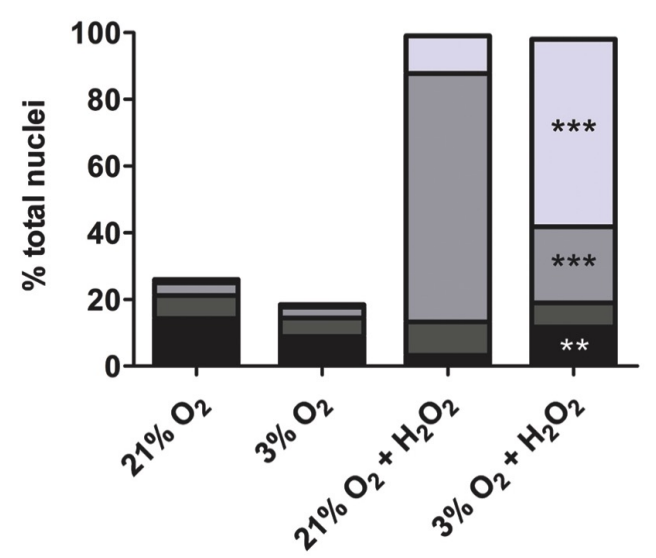

$\square$ Completely stained nuclei $\square 10$ to 20 spots $\square$ More than 20 spots

1 to 10 spots 
$589 \quad$ Figure 8

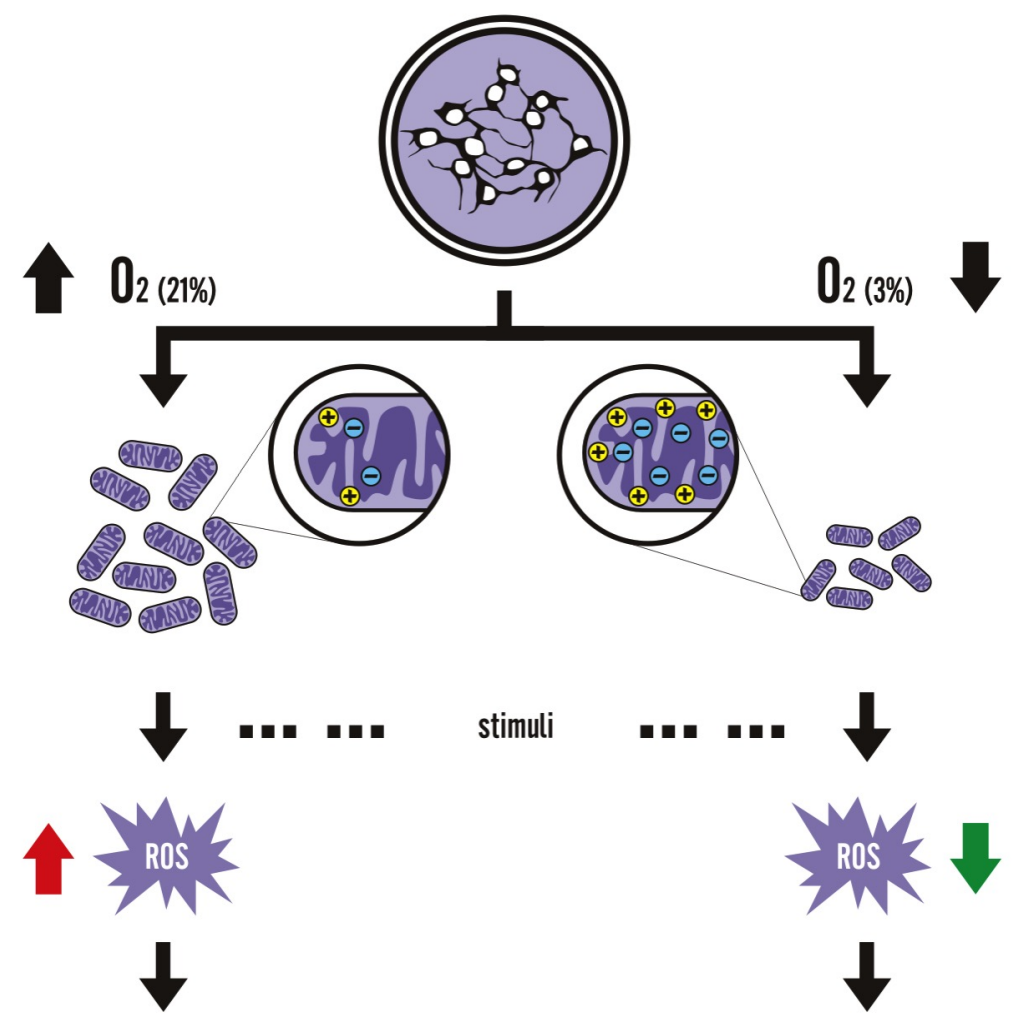

GPx Activity

$2 \mathrm{GSH}+\mathrm{H}_{2} \mathrm{O}_{2} \Rightarrow \mathrm{GSSG}+2 \mathrm{H}_{2} \mathrm{O}$

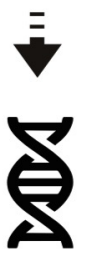

GPx Activity

$2 \mathrm{GSH}+\mathrm{H}_{2} \mathrm{O}_{2} \rightarrow \mathrm{GSSG}+2 \mathrm{H}_{2} \mathrm{O}$

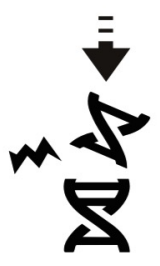


591 Supplemental information 1.

act.Casp3/7

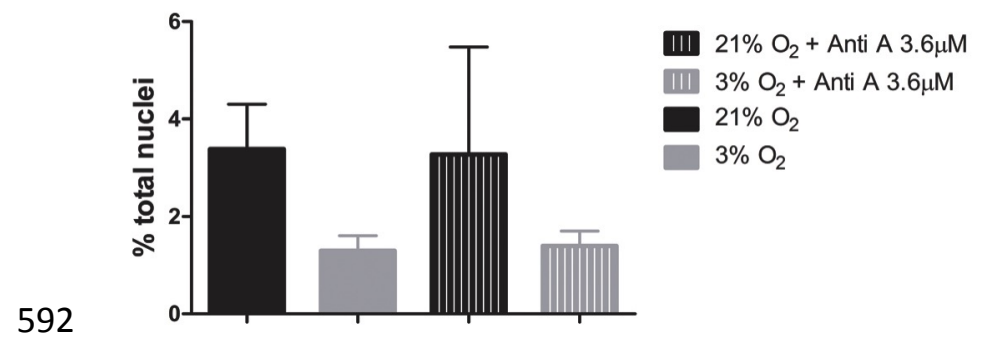


593 Supplemental information 2.

A

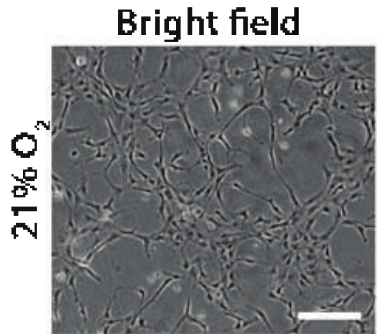

PSA-NCAM/DAPI
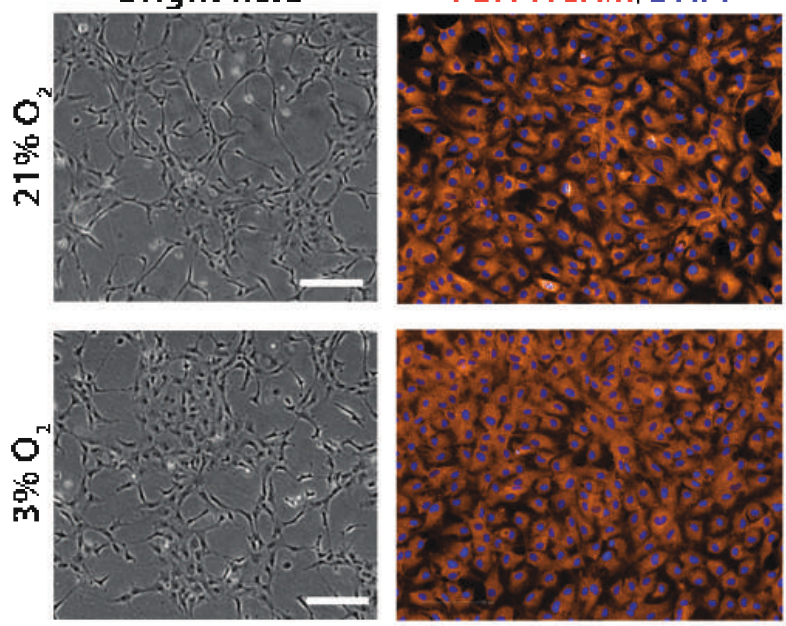

B
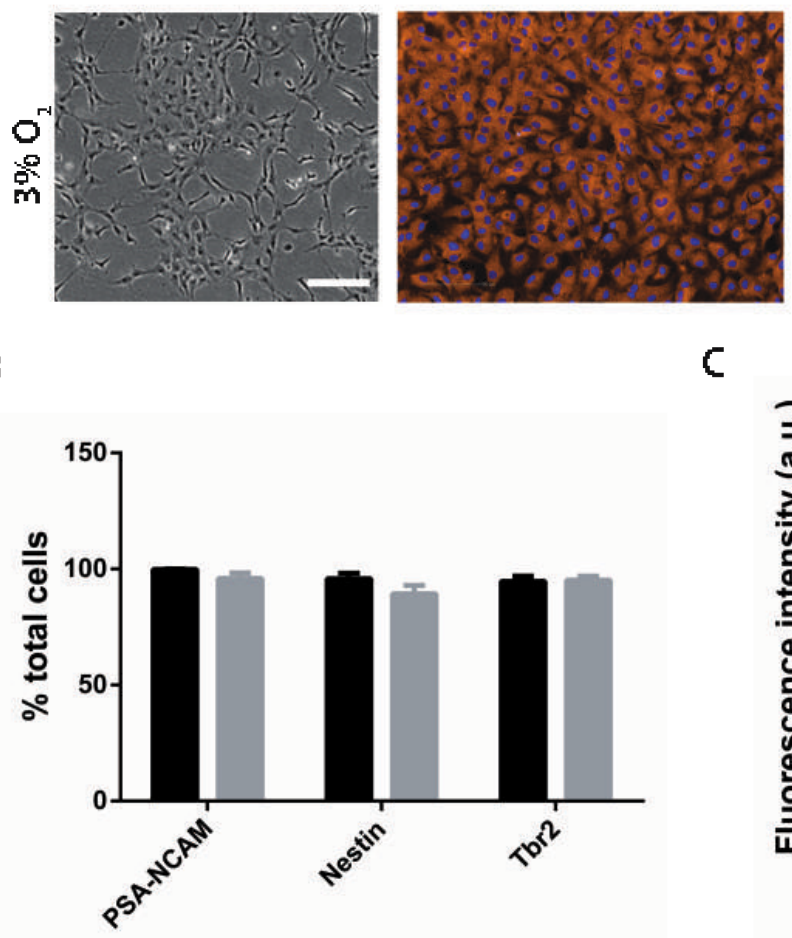

Nestin/DAPI
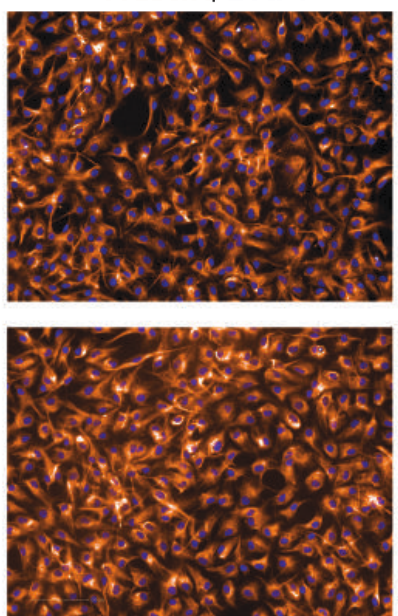

C
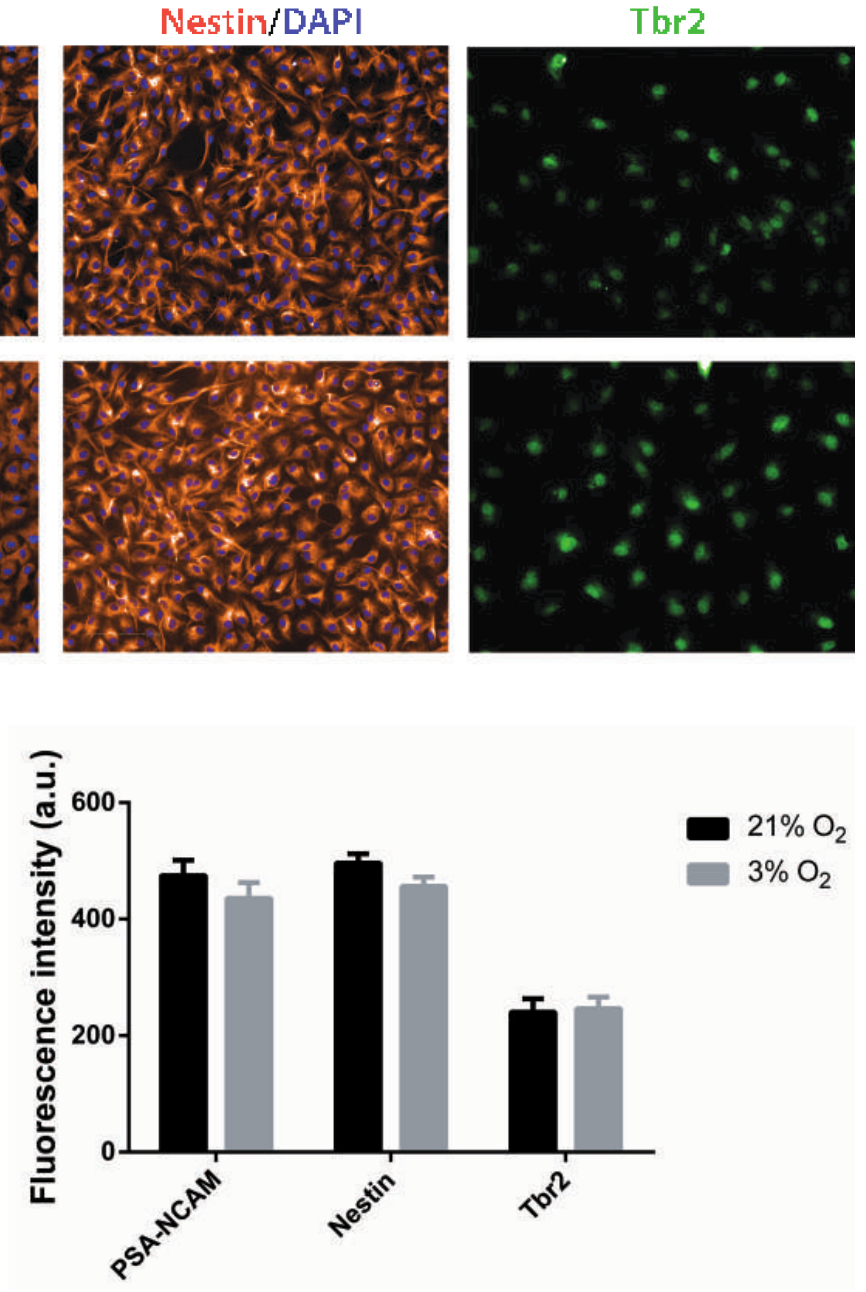